\title{
Sulfur-Doped Carbons from Durian Peels, Their Surface Characteristics, and Electrochemical Behaviors
}

\author{
Susilo Sudarman Desa, Takafumi Ishii, and Khanin Nueangnoraj*
}

Cite This: ACS Omega 2021, 6, 24902-24909

Read Online

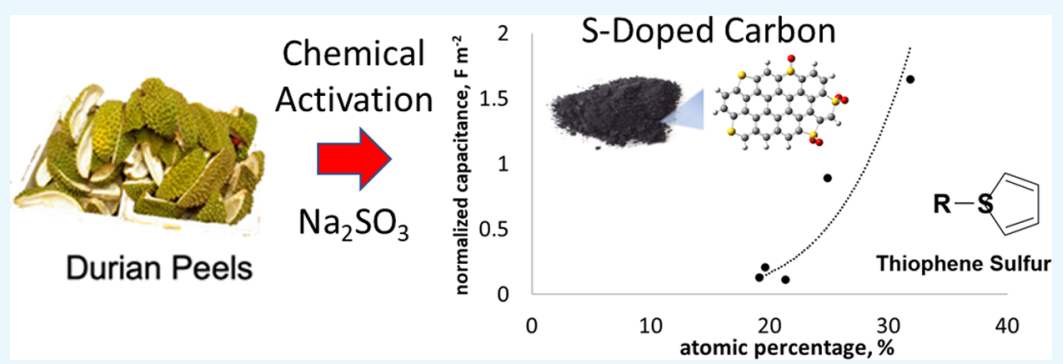

ABSTRACT: Durian peels are an agricultural waste in Asian countries, including Thailand, Indonesia, and Malaysia, which can be used as a precursor for the production of activated carbon. The objective of this work is to produce activated carbon from durian peels by chemical activation using sodium sulfite $\left(\mathrm{Na}_{2} \mathrm{SO}_{3}\right)$ as an activating and sulfur-doping agent. The process parameter investigated in this study was the activation temperature $\left(500-900{ }^{\circ} \mathrm{C}\right.$ ) at a fixed impregnation ratio (durian to activating agent of $1: 1$, by weight). Specific surface areas and pore structures were determined by nitrogen adsorption and desorption measurements, and elemental compositions were characterized by CHNSO analysis. The chemical structure and surface functionality were examined by X-ray photoelectron spectroscopy. The electrochemical behavior of the obtained activated carbon was characterized in $6 \mathrm{M} \mathrm{KOH}$ using a three-electrode configuration. It was found that the sulfur content decreases with activation temperature. In contrast, the specific surface area of the activated carbon increases with activation temperature. However, the sample activated at 900 ${ }^{\circ} \mathrm{C}$ with the highest specific surface area $\left(1499 \mathrm{~m}^{2} \mathrm{~g}^{-1}\right)$ has a lower specific capacitance $\left(166 \mathrm{~F} \mathrm{~g}^{-1}\right)$ than the one activated at $700{ }^{\circ} \mathrm{C}$ $\left(183 \mathrm{~F} \mathrm{~g}^{-1}\right)$. This could be due to the presence of a pseudocapacitance caused by the organic sulfur functional groups such as thiophene, sulfone, and sulfoxide, which can trigger a surface redox reaction, leading to a higher capacitance.

\section{INTRODUCTION}

There are many materials that can be used as adsorbents in gas adsorption and wastewater treatment, and the most commonly used adsorbents are activated carbons (ACs). ACs are carbonaceous materials that have high porosity, surface area, and surface functional groups. ACs can be widely used not only in gas adsorption and wastewater treatment but also in numerous other applications such as catalyst supports, gas separation and storage, solvent recovery and decolorization, and an electrode for supercapacitors.

ACs can be produced from various precursors, which are generally low-cost biomass. The precursors for the preparation of AC usually contain carbon, such as coals ${ }^{1,2}$ and coconut shells. ${ }^{3}$ Their adsorption capacity, wettability, and ion storage capacity can be enhanced by introducing heteroatoms such as nitrogen, oxygen, and sulfur into the carbon skeleton. ${ }^{4-7}$ Heteroatoms are usually introduced into the carbon framework of ACs by the following approaches: (i) post-treatment of ACs by chemical reactions with reagents containing the desired heteroatoms, ${ }^{8}$ (ii) molecular grafting of heteroatoms onto the carbon framework, ${ }^{9}$ and (iii) carbonization and activation of heteroatom-rich carbonaceous materials. ${ }^{10}$ Chemical impreg- nation with a chemical agent such as $\mathrm{ZnCl}_{2}, \mathrm{H}_{3} \mathrm{PO}_{4}$, and $\mathrm{KOH}$ can prevent tar formation and enhance carbon conversion. In addition, certain chemical agents can increase certain functional groups in ACs (heteroatom-doped carbon). ${ }^{11-13}$

There are numerous reports on $\mathrm{AC}$ preparation from biomass waste, but the uses of durian peels have rarely been investigated. It is desirable to develop the effective utilization of such biomass as it is cost-effective. Durian peels are one of the most abundant biomasses from agriculture in Thailand, Indonesia, and Malaysia. They naturally contain about $60 \%$ carbon but low sulfur content $(0.1 \%) .{ }^{14}$ Sulfur-containing compounds such as $\mathrm{Na}_{2} \mathrm{O}_{3} \mathrm{~S}_{2}, \mathrm{Na}_{2} \mathrm{SO}_{4}$, and $\mathrm{H}_{2} \mathrm{SO}_{4}$ have been used as activating agents to incorporate sulfur into the carbon frameworks. ${ }^{15-19}$ However, chemical activation with sodium sulfite $\left(\mathrm{Na}_{2} \mathrm{SO}_{3}\right)$ has never been reported to our knowledge.

Received: July 15, 2021

Published: September 16, 2021 
Table 1. Carbon, Hydrogen, and Nitrogen Contents of the Raw Materials and the As-Prepared ACs

\begin{tabular}{|c|c|c|c|c|c|c|c|c|c|}
\hline \multirow[b]{2}{*}{ sample } & \multirow[b]{2}{*}{$\mathrm{C}\left(\% \mathrm{w} \mathrm{w} \mathrm{w}^{-1}\right)$} & \multicolumn{3}{|c|}{ elemental composition } & \multirow[b]{2}{*}{$\mathrm{S} / \mathrm{C}$} & \multirow[b]{2}{*}{$S_{\mathrm{BET}}\left(\mathrm{m}^{2} \mathrm{~g}^{-1}\right)$} & \multirow[b]{2}{*}{$V_{\text {total }}\left(\mathrm{cm}^{3} \mathrm{~g}^{-1}\right)$} & \multirow[b]{2}{*}{$V_{\text {micro }}\left(\mathrm{cm}^{3} \mathrm{~g}^{-1}\right)$} & \multirow[b]{2}{*}{$V_{\text {meso }}\left(\mathrm{cm}^{3} \mathrm{~g}^{-1}\right)$} \\
\hline & & $\mathrm{H}\left(\% \mathrm{w} \mathrm{w}^{-1}\right)$ & $\mathrm{N}\left(\% \mathrm{w} \mathrm{w}^{-1}\right)$ & $\mathrm{S}\left(\% \mathrm{w} \mathrm{w}^{-1}\right)$ & & & & & \\
\hline DP & 43.64 & 6.32 & 0.78 & 0.19 & 0.0043 & & & & \\
\hline DP900 & 74.67 & 1.62 & 1.44 & 0.04 & 0.0005 & 4.36 & 0.062 & 0.002 & 0.060 \\
\hline AC500 & 51.23 & 2.84 & 0.82 & 17.55 & 0.3426 & 24 & 0.23 & 0.01 & 0.22 \\
\hline AC600 & 59.59 & 2.80 & 1.07 & 11.19 & 0.1878 & 133 & 0.30 & 0.06 & 0.24 \\
\hline AC700 & 71.08 & 2.77 & 0.93 & 2.20 & 0.0309 & 852 & 0.75 & 0.33 & 0.42 \\
\hline AC800 & 79.12 & 2.62 & 0.87 & 1.86 & 0.0235 & 1279 & 1.09 & 0.47 & 0.62 \\
\hline AC900 & 73.56 & 5.78 & 1.35 & 0.65 & 0.0088 & 1499 & 1.19 & 0.56 & 0.63 \\
\hline
\end{tabular}

In this work, S-doped activated carbon is prepared from durian peels using sodium sulfite $\left(\mathrm{Na}_{2} \mathrm{SO}_{3}\right)$ as an activating agent. The aim of this study is to investigate the effects of activation temperature on the sulfur content of ACs as well as their surface characteristics. Moreover, the electrochemical behavior of the obtained ACs will be investigated. It was found that the sulfur content can decrease the specific surface area of the ACs. Interestingly, the specific capacitance does not depend on the specific surface area of the ACs. Therefore, it is suggested that sulfur would play an important role in the electrochemical capacitance of electrodes made of S-doped activated carbon. Although there are many methods to synthesize sulfur-doped carbon from wasted biomass such one-pot synthesis and post-treatment synthesis and $\mathrm{H}_{2} \mathrm{SO}_{4}$ have been used to incorporate sulfur with the carbon framework, it unfortunately requires post-treatment to create the porosity. ${ }^{17,18}$ In addition, $\mathrm{H}_{2} \mathrm{SO}_{4}$ is a strong acid, and the process involved inevitably suffers from corrosion. Instead, saltcontaining sulfur, such as $\mathrm{Na}_{2} \mathrm{SO}_{4}, \mathrm{Na}_{2} \mathrm{SO}_{3}$, and $\mathrm{Na}_{2} \mathrm{O}_{3} \mathrm{~S}_{2}$, can be used as a sulfur source where the etching effect would play a role in creating the porosity. ${ }^{16}$ Beneficially, sulfur-doped carbon prepared with $\mathrm{Na}_{2} \mathrm{SO}_{3}$ is easy to handle without any pre- or post-treatment as using $\mathrm{Na}_{2} \mathrm{O}_{3} \mathrm{~S}_{2}{ }^{15}$ and $\mathrm{K}_{2} \mathrm{SO}_{4}{ }^{19}$ Although the price of $\mathrm{Na}_{2} \mathrm{SO}_{3}$ is somewhat higher than that of $\mathrm{H}_{2} \mathrm{SO}_{4}$ (as its usage is still limited to food preservative and antioxidant), taking the aforementioned advantages as well as the abundant amount of raw materials used into consideration, this work could realistically be applied commercially.

\section{RESULTS AND DISCUSSION}

2.1. Elemental Analysis. The elemental compositions of the raw materials (dried durian peels, DP) and the obtained ACs, including carbon, hydrogen, nitrogen, and sulfur, are listed in Table 1 . Compared with the sulfur content of the raw material (DP, $0.19 \% \mathrm{w} / \mathrm{w})$, the sulfur content of all ACs is drastically increased. By increasing the activation temperature, the carbon content of the sample is increased up to the optimum at $800{ }^{\circ} \mathrm{C}$ and then slightly decreased. However, the sulfur content decreases exponentially with increasing activation temperature. This can be clearly seen in Figure 1 when considering $\mathrm{S} / \mathrm{C}$ as a function of activation temperature. These trends are common as a result of many reactions at high temperatures such as dehydrogenation, desulfurization, and condensation. $^{20}$ This relationship shows that sulfur content could be decomposed at high temperatures as COS, $\mathrm{SO}_{2}, \mathrm{H}_{2} \mathrm{~S}$, and $\mathrm{CS}_{2}{ }^{21,22}$

2.2. Porosity Analysis. $\mathrm{N}_{2}$-sorption measurement was employed to characterize the porosity of ACs. Figure 2a reveals that DP900 and AC500 adsorb deficient nitrogen gas, indicating the nonporosity of the material. This result is also confirmed in Table 1, which shows that these two samples only

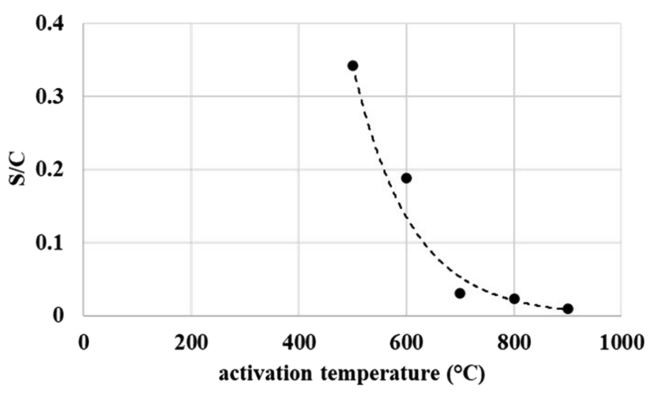

Figure 1. Relation between the sulfur content (S/C) of the ACs and the activation temperature.

consist of less than $25 \mathrm{~m}^{2} \mathrm{~g}^{-1} S_{\mathrm{BET}}$. The pore size distribution calculated by the NLDFT method in Figure $2 \mathrm{~b}$ shows a plateau line for these samples (DP900 and AC500). When the activation temperature is increased, the sorption isotherm transitions to the IV type, indicating the combination of mesopore and micropore structures. ${ }^{23}$ The hysteresis loops in the range of relative pressure of $0.4-1.0$ become more evident by increasing the activation temperature, indicating the abundance of mesopore structures. ${ }^{24}$ These results are also confirmed by the pore size distribution shown in Figure $2 \mathrm{~b}$. As listed in Table 1, AC900 has the highest porosity in terms of $S_{\mathrm{BET}}, V_{\text {total }}, V_{\text {micro, }}$ and $V_{\text {meso }}$. Decreasing the activation temperature leads to a decrease in the surface area and pore volume of the samples.

The relationship between the activation temperature and the pore characteristic of ACs is shown in Figure 3a,b. As the activation temperature increases, the surface area and total pore volume increase linearly. As shown in Figure 3b, the total pore volume is dominated by mesopores at low activation temperature. At higher activation temperature, more micropores are formed so that the micropore volume and mesopore volume are almost equal. In contrast, the surface area decreases exponentially with the $\mathrm{S} / \mathrm{C}$ value (Figure $3 \mathrm{c}$ ), suggesting that sulfur in the carbon framework could collapse the structure or enlarge the pores, leading to a decrease in the specific surface area. ${ }^{17,25,26}$ This explanation is also supported by the fact that the sample activated at $500{ }^{\circ} \mathrm{C}$ (the highest $\mathrm{S} / \mathrm{C}$ value) is dominated by mesopores, as shown in Figure $3 \mathrm{~d}$. Moreover, volatile sulfur could be evaporated at high activation temperatures, resulting in a more ordered and condensed carbon structure, leading to the formation of micropores. ${ }^{27}$

2.3. Surface Characteristics. The surface functionality of the obtained ACs was investigated by FTIR spectroscopy (Figure S1). The spectra show several vibrations including $\mathrm{C}=\mathrm{C}$ (aromatic, $\left.1580-1615 \mathrm{~cm}^{-1}\right), \mathrm{SO}_{2}$ symmetric stretching $\left(1120-1190 \mathrm{~cm}^{-1}\right), \mathrm{S}=\mathrm{O}$ stretching $\left(1020-1060 \mathrm{~cm}^{-1}\right), \mathrm{C}-$ $S$ stretching $\left(600-700 \mathrm{~cm}^{-1}\right)$, and $S-S$ stretching $(450-550$ 

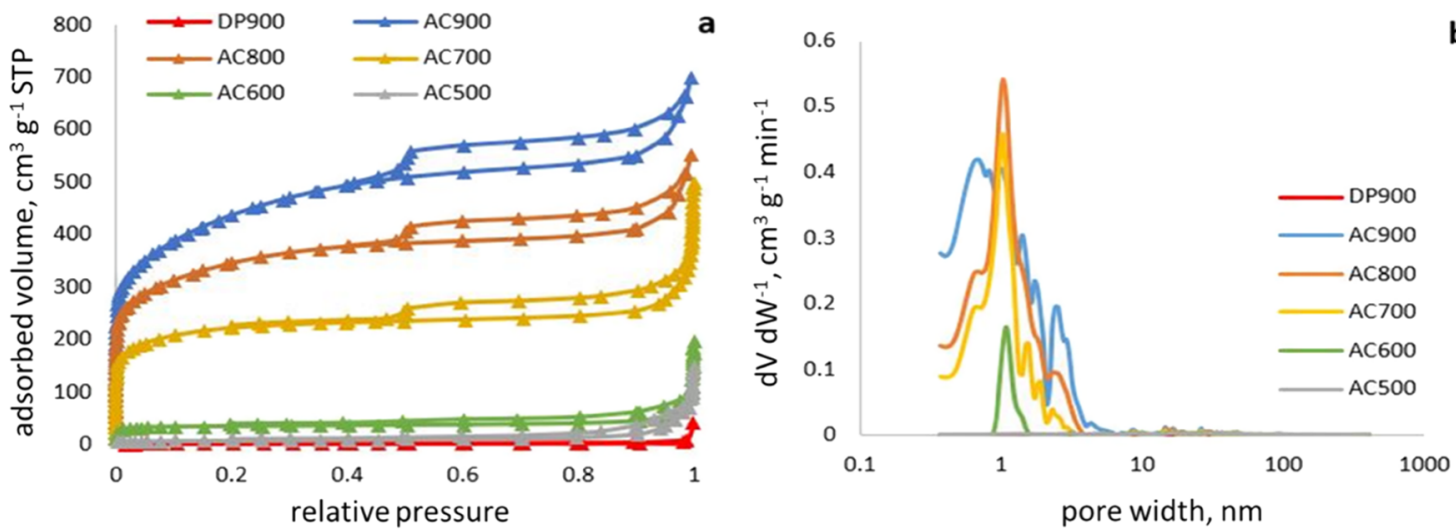

Figure 2. (a) Nitrogen-sorption isotherms at $-196^{\circ} \mathrm{C}$ and (b) DFT pore size distributions of the obtained ACs.

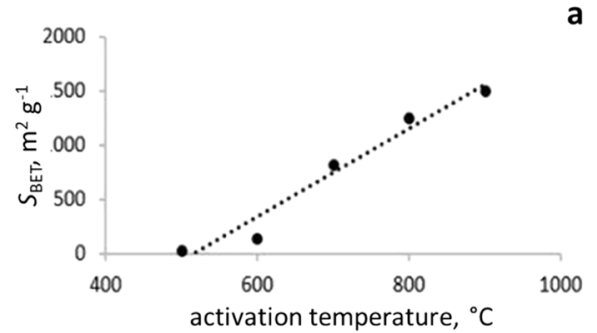

a
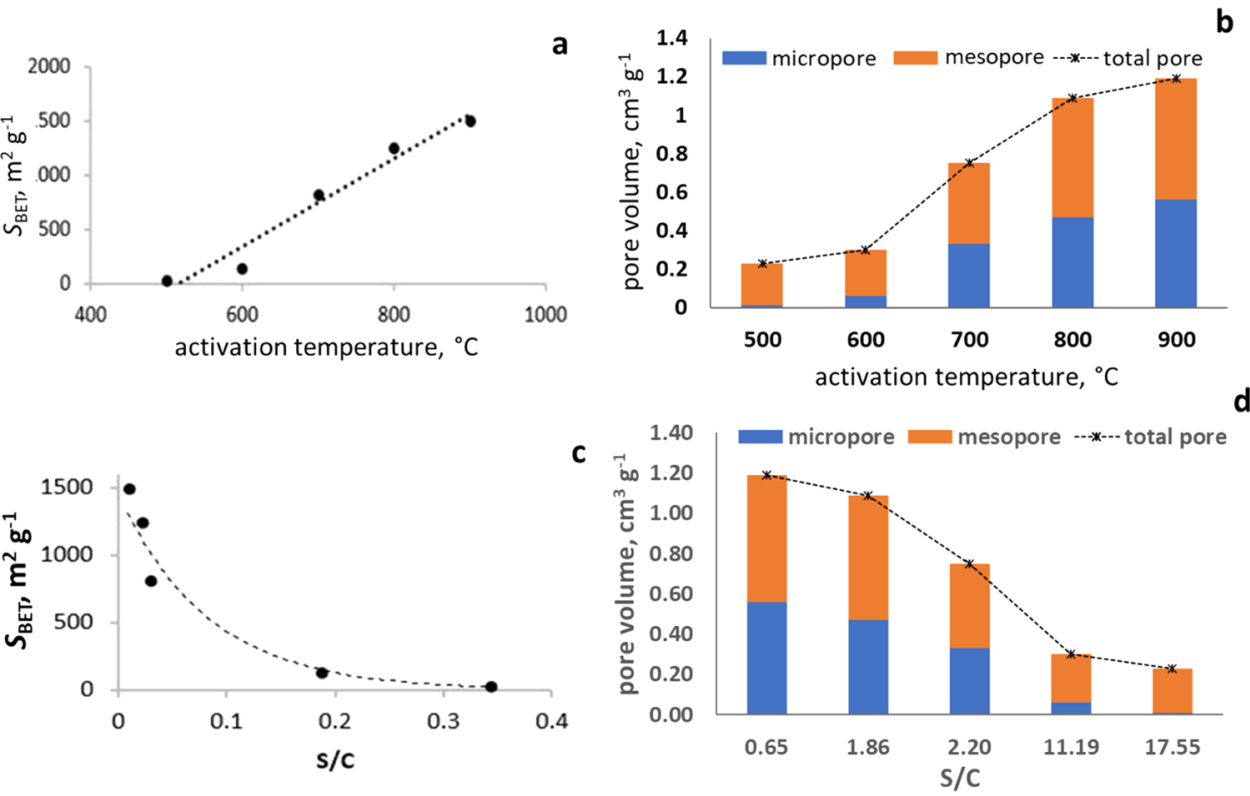

Figure 3. (a) Dependence of the specific surface area on the activation temperature, (b) effect of activation temperature on the formation of pore size, and $(c, d)$ the relations of S/C to the specific surface area and pore volume, respectively.

$\left.\mathrm{cm}^{-1}\right){ }^{28,29}$ Further investigation on the surface functionality has been done by XPS, and their S $2 p$ spectra are shown in Figure 4. Based on the deconvolution using the Gaussian equation, each $\mathrm{AC}$ consists of sulfur functional groups in the form of mercaptan $\left(\mathrm{C}-\mathrm{SH}_{3}, 161.2-163.6 \mathrm{eV}\right)$, thiophene $(\mathrm{C}-$ $\mathrm{S}-\mathrm{C}, 164.0-164.4 \mathrm{eV})$, sulfoxide (C-SO-C, 165.0-166.0 $\mathrm{eV}$ ), sulfone $\left(\mathrm{C}-\mathrm{SO}_{2}-\mathrm{C}, 167.0-168.3 \mathrm{eV}\right)$, and inorganic sulfur $(168.4-175.0 \mathrm{eV}) .^{30-32}$ The atomic percentage estimated from the peak area after the deconvolution of each sulfur functional group is listed in Table 2. With the exception of DP900, all samples contain a small amount of mercaptan (less than 3.5\%), while the content of thiophene, sulfoxide, and sulfone varies from 10 to $35 \%$.

To understand the change of the surface functional group during activation, the relationship between the atomic percentage of each sulfur functional group and the activation temperature is established, as shown in Figure 5. However, the chemical transformations of the sulfur surface functional group during pyrolysis are still a complex issue. Mercaptan, which combines aliphatic $\mathrm{C}$ with $\mathrm{S}$, has a lower content at each activation temperature. This is the cleavage of $\mathrm{S}-\mathrm{C}$ at low temperatures to form $S$ radicals $(\cdot S \cdot)$, which further react with carbon sources to form other sulfur functional groups. ${ }^{21}$ If the activation temperature is increased up to $700{ }^{\circ} \mathrm{C}$, the thiophene content decreases to a certain extent and then remains constant. This could be due to the presence of unstable thiol groups, which transform into a stable thiophene structure at high temperatures. ${ }^{33,34}$ The sulfone content decreases to the minimum at an activation temperature of $700{ }^{\circ} \mathrm{C}$, which is due to the thermal reduction of the sulfone to a sulfoxide group, so that the sulfoxide content increases up to $700{ }^{\circ} \mathrm{C}$. Another possible explanation for the unpredictable thermal decomposition of the sulfone is due to the chemical neighborhood of the sulfone. Sulfone with aliphatic carbon can decompose at low temperatures, while sulfone with cyclic carbon can decompose at the temperatures up to $500{ }^{\circ} \mathrm{C} .{ }^{35}$ In addition, thiophene and oxygen-containing sulfur functional groups (sulfone and sulfoxide) can decompose as $\mathrm{H}_{2} \mathrm{~S}, \mathrm{SO}_{2}$, or COS, which could further reduce the sulfur content. ${ }^{21,36}$

2.4. Electrochemical Capacitive Behaviors. The electrochemical behavior of the obtained ACs was investigated using cyclic voltammetry $\left(\mathrm{CV}\right.$, a sweep rate of $\left.1 \mathrm{mV} \mathrm{s}^{-1}\right)$ and galvanostatic charge-discharge measurement (GCD, a current density of $\left.1 \mathrm{~A} \mathrm{~g}^{-1}\right)$. As shown in Figure $6 \mathrm{a}, \mathrm{b}$, the 

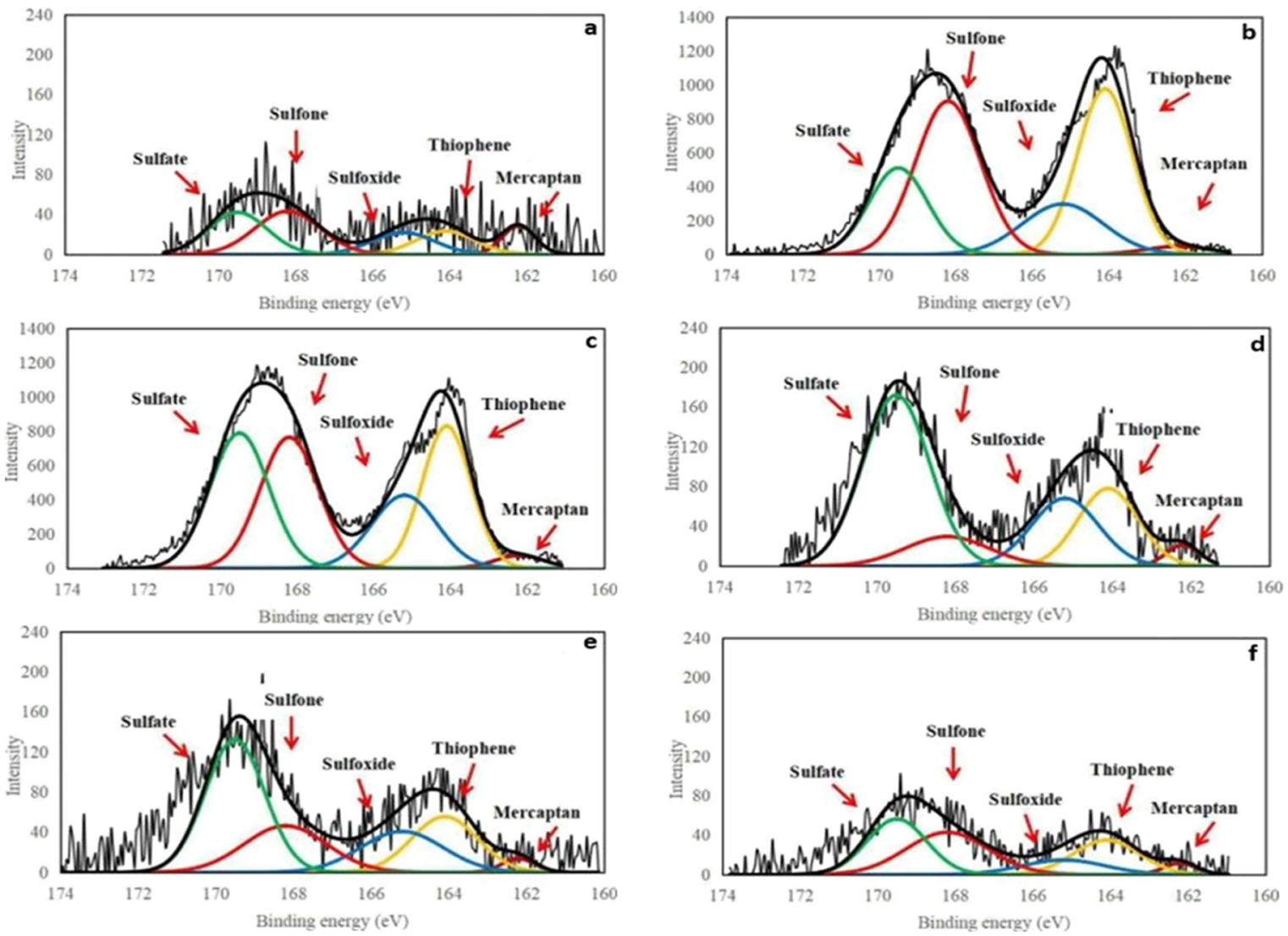

Figure 4. S 2p spectra of (a) DP900, (b) AC500, (c) AC600, (d) AC700, (e) AC800, and (f) AC900 samples.

Table 2. Atomic Percentages of Each Sulfur Functionality Estimated from XPS Analysis

\begin{tabular}{lccccc} 
& \multicolumn{5}{c}{ atomic percentage (\%) } \\
\cline { 2 - 6 } sample & mercaptan & thiophene & sulfoxide & sulfone & $\begin{array}{c}\text { inorganic sulfur/ } \\
\text { sulfate }\end{array}$ \\
DP900 & 10.49 & 15.29 & 15.25 & 31.50 & 27.47 \\
AC500 & 1.71 & 31.82 & 14.23 & 34.65 & 17.59 \\
AC600 & 2.11 & 24.91 & 17.02 & 27.54 & 28.42 \\
AC700 & 2.87 & 19.63 & 19.06 & 10.32 & 48.12 \\
AC800 & 2.34 & 19.12 & 17.54 & 19.94 & 41.06 \\
AC900 & 3.50 & 21.37 & 11.33 & 32.75 & 31.05 \\
\hline
\end{tabular}

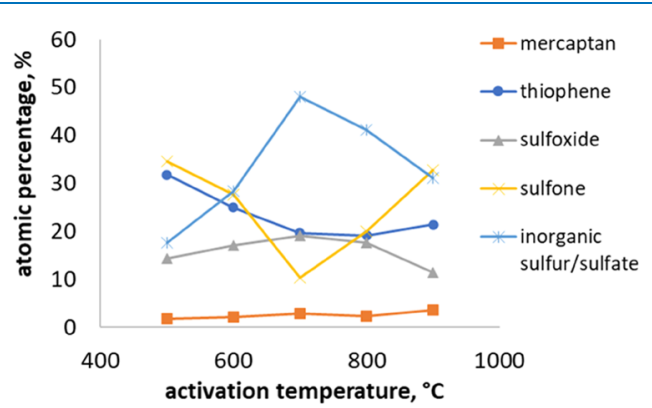

Figure 5. Correlation of each sulfur functional group to the activation temperature.

voltammograms of the ACs (except AC500) have a quasirectangular shape and GCD straight discharge lines typical of the EDLC mechanism. However, AC, which was activated at $500{ }^{\circ} \mathrm{C}$, shows low $\mathrm{CV}$ and GCD performances due to its low electrical conductivity, consistent with a large IR drop in the initial discharge curve. Cyclic voltammograms at various scan rates, galvanostatic charge/discharge curves at various current densities, and specific capacitance as a function of current density of the obtained ACs can be found in the Supporting Information (Figures S2-S4).

According to the results of $\mathrm{CV}$ and GCD, the specific capacitance of ACs was estimated as listed in Table 3. AC700 exhibits the highest specific capacitance of $183 \mathrm{~F} \mathrm{~g}^{-1}$, which is comparable to those in the literature using the same electrolyte (Table S1), 15,17,33,37 and decreases slightly at higher activation temperature. Considering in terms of specific energy and power (Figure S5), the obtained S-doped ACs still show compatible performances to those reported in the literature. $^{15,17,37}$ Moreover, the obtained ACs exhibit excellent capacitance retention after 1000 cycles of the cyclability test (Figure S6). To understand the effects of surface functional groups on the charge storage capacity, the normalized capacitance is considered by dividing the specific capacitance by the specific surface area. As can be seen in Table 3, AC500 drives an excellent normalized capacitance $\left(1.65 \mathrm{~F} \mathrm{~m}^{-2}\right)$, which is higher than the others. This could be due to the highest sulfur content of this sample with the lowest specific surface area. However, as mentioned earlier, this sample has low conductivity, which could hinder the ion transport of the electrode. Moreover, the normalized capacitance decreases at high activation temperature when the sulfur content decreases as aforementioned (Figure 7a). This clearly indicates the role of sulfur in altering the surface properties and charge storage capability of ACs.

The relationship between normalized capacitance and the atomic percentage of each sulfur functional group is shown in 

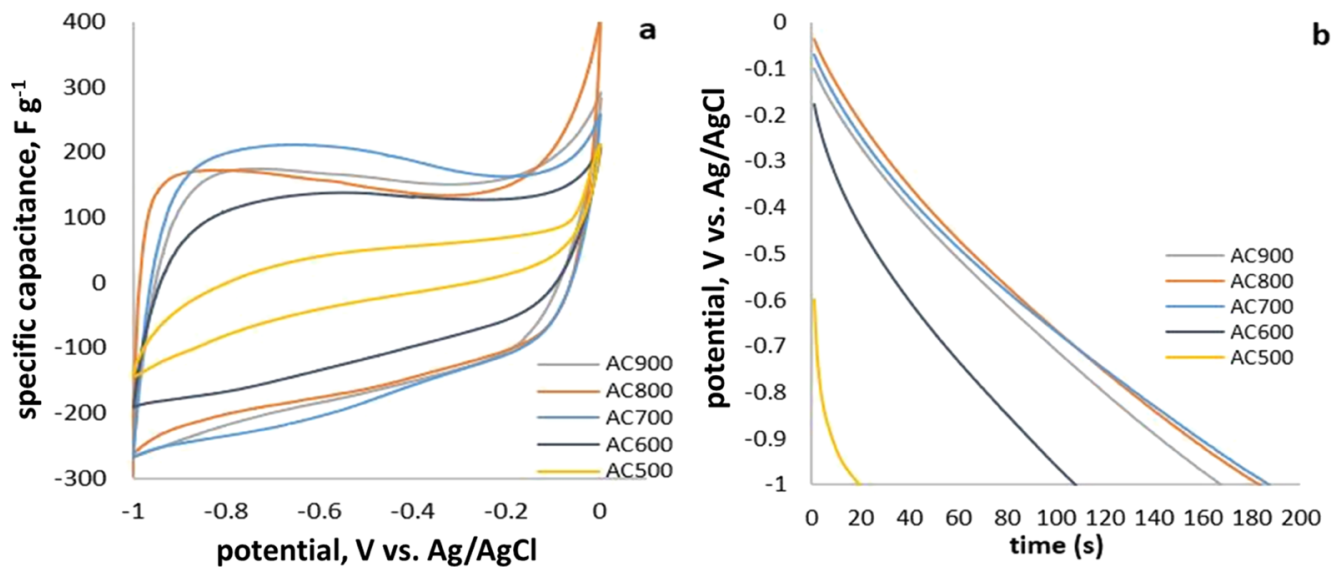

Figure 6. (a) Cyclic voltammograms and (b) discharge curves of the obtained ACs. The scan rate and current density were $5 \mathrm{mV} \mathrm{s}^{-1}$ and $1 \mathrm{~A} \mathrm{~g}{ }^{-1}$, respectively, using a three-electrode configuration in $6 \mathrm{M} \mathrm{KOH}$.

Table 3. Specific Capacitance and Normalized Capacitance of the Obtained ACs

$\begin{array}{ccc}\text { sample } & \text { specific capacitance }\left(\mathrm{F} \mathrm{g}^{-1}\right) & \text { normalized capacitance }\left(\mathrm{F} \mathrm{m}^{-2}\right) \\ \text { AC500 } & 41 & 1.65 \\ \text { AC600 } & 118 & 0.89 \\ \text { AC700 } & 183 & 0.21 \\ \text { AC800 } & 170 & 0.13 \\ \text { AC900 } & 166 & 0.11\end{array}$

Figure $7 \mathrm{~b}-\mathrm{f}$. Although it can be seen in Figure $7 \mathrm{~b}$ that mercaptan-sulfur has a negative effect on the normalized capacitance, its content is 10 times lower than those of the other functional groups (see Figure 5). Therefore, these effects could be reasonably negligible in this work.

It can be seen that thiophene (Figure 7c) and inorganic sulfur (or sulfate, Figure $7 \mathrm{f}$ ) could play a significant role in normalized capacitance, while sulfoxide (Figure $7 \mathrm{~d}$ ) and sulfone (Figure $7 \mathrm{e}$ ) do not seem to have a clear tendency. As shown in Figure $7 c$, thiophene sulfur has a positive impact on normalized capacitance. Although sulfone and sulfoxide species are expected to play a positive role in the overall capacitance by undergoing faradic redox reactions, ${ }^{38}$ it has been reported that thiophenic-like sulfur can also contribute to the pseudocapacitance. ${ }^{3,39}$ Moreover, sulfur can induce structural defects in the carbon framework due to its relatively
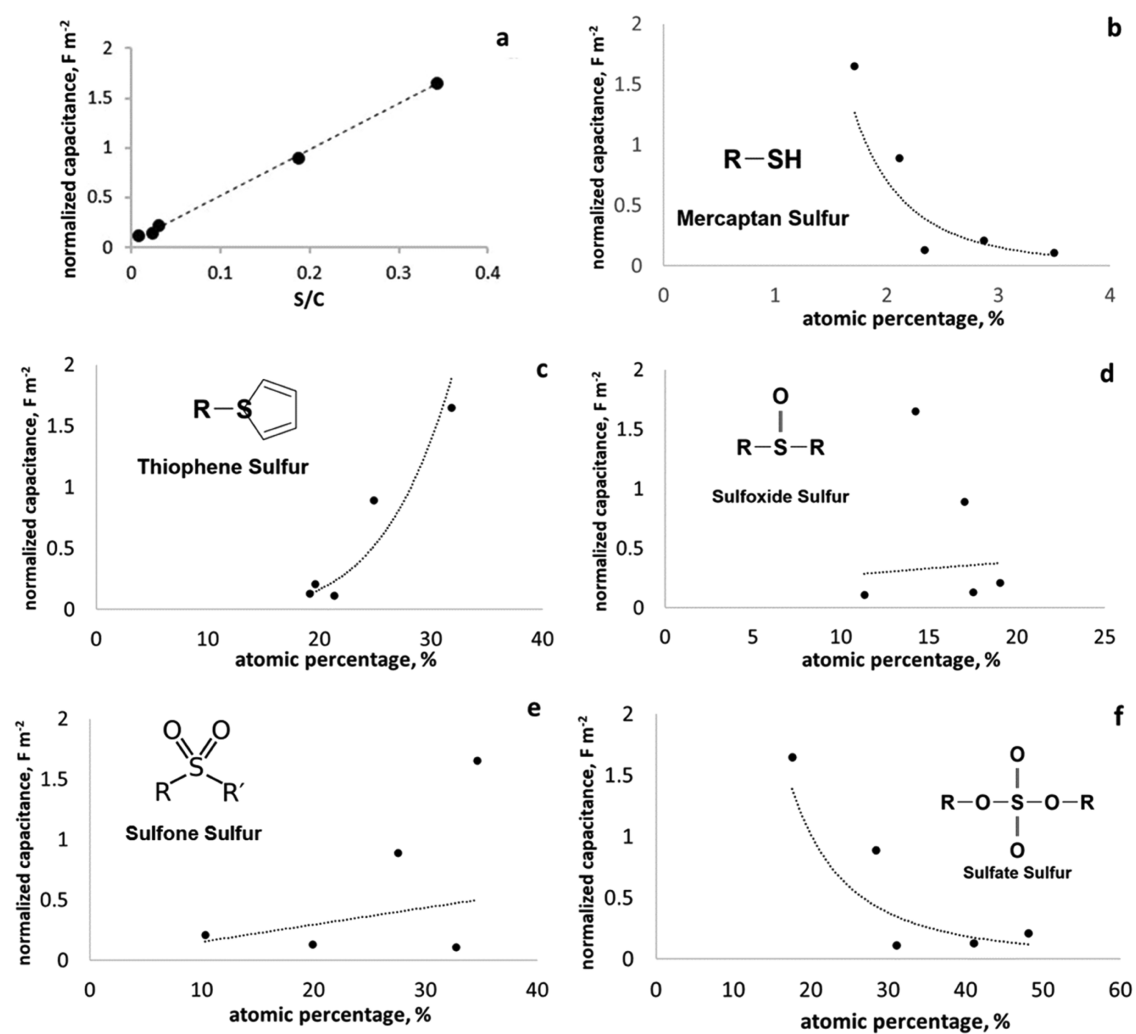

Figure 7. Relations of (a) normalized capacitance versus S/C and $(b-f)$ normalized capacitance versus atomic percentage of each sulfur functional group. 
large covalent radius, resulting in more active sites for charge localization. $^{40}$ In addition, sulfur doping could alter the electronic density of the carbonaceous material, and sulfur possesses a lone pair, leading to an increase in the reactivity of ACs. ${ }^{15,41}$ This further supports and highlights the use of Sdoped AC as an electrode in electrochemical energy storage devices.

\section{CONCLUSIONS}

In conclusion, S-doped activated carbon was successfully synthesized from durian peels by chemical activation with a mild activating agent, $\mathrm{Na}_{2} \mathrm{SO}_{3}$. The results indicate that the sulfur content plays an important role in the specific surface area and the formation of the microporous structure of ACs. AC700 exhibits the highest capacitance of $183 \mathrm{~F} \mathrm{~g}^{-1}$ at $5 \mathrm{mV}$ $\mathrm{s}^{-1}$. Nevertheless, AC500 with the highest sulfur content can store a large amount of charge even at a low $S_{\mathrm{BET}}$. It is suggested that the presence of thiophene species could contribute to increase the charge storage capacity of S-doped AC.

\section{MATERIALS AND METHODS}

4.1. Preparation of Activated Carbons. Durian peels (DP) were obtained from a local market (Talaad Thai, Pathum Thani province, Thailand). It was washed and dried at $105{ }^{\circ} \mathrm{C}$ for $24 \mathrm{~h}$. It was then ground into powder form and impregnated with the activating agent, $\mathrm{Na}_{2} \mathrm{SO}_{3}$ (weight ratio $1: 1)$. After that, the impregnated sample was placed in an alumina boat and inserted into a horizontal tube furnace. The furnace was heated to $500-900{ }^{\circ} \mathrm{C}\left(5^{\circ} \mathrm{C} \mathrm{min}{ }^{-1}\right)$ under a nitrogen atmosphere and held for $1 \mathrm{~h}$. After the carbonization/ activation, it was cooled to room temperature under a $\mathrm{N}_{2}$ flow. The AC products were washed with $\mathrm{RO}$ water and dried at 110 ${ }^{\circ} \mathrm{C}$ for $24 \mathrm{~h}$. The samples were labeled as AC500, AC600, AC700, AC800, and AC900, depending on the activation temperature (500, 600, 700, 800, and $900{ }^{\circ} \mathrm{C}$, respectively). For comparison, the sample that was simply carbonized at 900 ${ }^{\circ} \mathrm{C}$ for $1 \mathrm{~h}$ (without $\mathrm{Na}_{2} \mathrm{SO}_{3}$ ) was labeled as DP900.

4.2. Characterization. The chemical composition of the dried durian peels and the obtained ACs was analyzed by elemental analysis (CHNSO analyzer, model: 628 series, Leco Corporation, USA). The specific surface area $\left(S_{\mathrm{BET}}\right)$ was calculated from the $\mathrm{N}_{2}$ adsorption isotherms at $77 \mathrm{~K}(-196$ ${ }^{\circ} \mathrm{C}$ ) using the Brunauer-Emmett-Teller (BET) equation. The total pore volume $\left(V_{\text {total }}\right)$ was estimated from the $\mathrm{N}_{2}$ adsorption amount at a relative pressure of 0.95 . The micropore volume $\left(V_{\text {micro }}\right)$ was calculated using the Dubinin-Radushkevich (DR) method. ${ }^{42}$ The mesopore volume $\left(V_{\text {meso }}\right)$ was determined by subtracting the micropore volume from the total pore volume. The pore size distribution was characterized by density functional theory (DFT). The chemical structure and surface functionality of the obtained ACs were examined by X-ray photoelectron spectroscopy (XPS) using $\mathrm{Mg} \mathrm{K} \alpha$ radiation (12 kV and $25 \mathrm{~mA}$ ). The obtained ACs were also characterized by Fourier transform infrared spectroscopy (FTIR, Nicole iS50, Thermo Fisher Scientific, $2.5 \mathrm{wt} \%$ in $\mathrm{KBr}$ ). The number of scans and resolution were 16 and 4, respectively.

Electrochemical characterizations were performed using a three-electrode configuration. To prepare the electrodes, the obtained ACs were mixed with carbon black and polytetrafluoroethylene (PTFE) in a weight ratio of 9:0.5:0.5 to form a uniform solid sheet. The sheet was then cut into a square shape and pressed into a stainless-steel mesh. After that, it was impregnated (overnight) with the electrolyte before the measurements. Cyclic voltammetry $(\mathrm{CV})$ and galvanostatic charge/discharge (GCD) were performed to observe the electrochemical behavior of the AC electrodes. The electrolyte, reference, and counter electrodes are $6 \mathrm{M} \mathrm{KOH}, \mathrm{Ag} / \mathrm{AgCl}$ in 3 $\mathrm{M} \mathrm{KCl}$, and a platinum rod, respectively. The specific capacitances $\left(\mathrm{F} \mathrm{g}^{-1}\right)$ were calculated from the integrated voltammogram using the following equation:

$$
C=\int_{E_{1}}^{E_{2}} \frac{i(E) \mathrm{d} E}{2\left(E_{2}-E_{1}\right) v m}
$$

where $\int_{E_{1}}^{E_{1}} i(E) \mathrm{d} E$ is the total charge resulting from the integration of the positive and negative sweep of the cyclic voltammogram $(\mathrm{C}), i(E)$ is the instantaneous current $(\mathrm{A}), E_{1}$ and $E_{2}$ are the cut-off potentials $(\mathrm{V}), m$ is the mass of active material in the samples $(\mathrm{g})$, and $v$ is the scan rate $\left(\mathrm{V} \mathrm{s}^{-1}\right)$. In addition, charge/discharge measurements were performed on the obtained ACs, and the resulting specific capacitance was calculated using the following equation:

$$
C=\frac{I}{\Delta V / \Delta t}
$$

where $C$ is the specific capacitance $\left(\mathrm{F} \mathrm{g}^{-1}\right), I$ is the current density, and $\Delta V / \Delta t$ is the slope of the charge/discharge plot (V $\left.\mathrm{s}^{-1}\right)$. The energy density $\left(E, \mathrm{Wh} \mathrm{kg}^{-1}\right)$ and power density $(P$, $\mathrm{W} \mathrm{kg}^{-1}$ ) (on an active mass) were calculated according to the following equations:

$$
\begin{aligned}
& E=\frac{1}{2} \cdot \frac{1}{4} \cdot \frac{1}{3.6} C V^{2} \\
& P=\frac{E}{t}
\end{aligned}
$$

where $V$ is the cell voltage after ohmic drop $(\mathrm{V})$ and $t$ is the discharge time $(\mathrm{h})$.

\section{ASSOCIATED CONTENT}

\section{Supporting Information}

The Supporting Information is available free of charge at https://pubs.acs.org/doi/10.1021/acsomega.1c03760.

FTIR spectra of the obtained ACs, cyclic voltammograms of the obtained ACs at various scan rates in $6 \mathrm{M}$ $\mathrm{KOH}$, galvanostatic charge/discharge curves of the obtained ACs at various current densities in $6 \mathrm{M}$ $\mathrm{KOH}$, specific capacitance of the obtained ACs as a function of current density in $6 \mathrm{M} \mathrm{KOH}$, comparison of several S-doped activated carbon in $6 \mathrm{M} \mathrm{KOH}$ electrolyte, Ragone plots of the obtained ACs, and cyclability test over 1000 cycles at $5 \mathrm{~A} \mathrm{~g}^{-1}$ in $6 \mathrm{M} \mathrm{KOH}$ (PDF)

\section{AUTHOR INFORMATION}

\section{Corresponding Author}

Khanin Nueangnoraj - Research Network of NANOTEC on NanoCatalysts and NanoMaterials for Sustainable Energy and Environment, School of Bio-Chemical Engineering and Technology, Sirindhorn International Institute of Technology, Thammasat University - Rangsit Campus, Pathum Thani 
12121, Thailand; ๑ orcid.org/0000-0002-6669-5007; Email: khanin@siit.tu.ac.th

\section{Authors}

Susilo Sudarman Desa - School of Bio-Chemical Engineering and Technology, Sirindhorn International Institute of Technology, Thammasat University - Rangsit Campus, Pathum Thani 12121, Thailand

Takafumi Ishii - International Research and Education Center for Element Science, Faculty of Science and Technology, Gunma University, Kiryu, Gunma 376-8515, Japan; @ orcid.org/0000-0001-8176-3699

Complete contact information is available at:

https://pubs.acs.org/10.1021/acsomega.1c03760

\section{Notes}

The authors declare no competing financial interest.

\section{ACKNOWLEDGMENTS}

This work was supported by Thammasat University Research Fund, Contact No. TUFT 053/2563 and the thesis support from the Scholarship for Excellent Foreign Student (EFS), Sirindhorn International Institute of Technology (SIIT), Thammasat University (TU). This work was also funded by National Research Council of Thailand (NRCT): N42A640324 and has been partially supported by the National Nanotechnology Center (NANOTEC), NSTDA, Ministry of Science and Technology, Thailand, through its program of Research Network of NANOTEC (RNN).

\section{REFERENCES}

(1) Teng, H.; Yeh, T.-S. Preparation of Activated Carbons from Bituminous Coals with Zinc Chloride Activation. Ind. Eng. Chem. Res. 1998, 37, 58-65.

(2) Lozano-Castelló, D.; Lillo-Ródenas, M. A.; Cazorla-Amorós, D.; Linares-Solano, A. Preparation of activated carbons from Spanish anthracite I Activation by KOH. Carbon 2001, 39, 741-749.

(3) Daud, W. M.; Ali, W. S. Comparison on pore development of activated carbon produced from palm shell and coconut shell. Bioresour. Technol. 2004, 93, 63-69.

(4) Mondal, A. K.; Kretschmer, K.; Zhao, Y.; Liu, H.; Fan, H.; Wang, G. Naturally nitrogen doped porous carbon derived from waste shrimp shells for high-performance lithium ion batteries and supercapacitors. Microporous Mesoporous Mater. 2017, 246, 72-80.

(5) Zhang, D.; Han, M.; Wang, B.; Li, Y.; Lei, L.; Wang, K.; Wang, Y.; Zhang, L.; Feng, H. Superior supercapacitors based on nitrogen and sulfur co-doped hierarchical porous carbon: Excellent rate capability and cycle stability. J. Power Sources 2017, 358, 112-120.

(6) Chen, F.; Yang, J.; Bai, T.; Long, B.; Zhou, X. Biomass wastederived honeycomb-like nitrogen and oxygen dual-doped porous carbon for high performance lithium-sulfur batteries. Electrochim. Acta 2016, 192, 99-109.

(7) Treeweranuwat, P.; Boonyoung, P.; Chareonpanich, M.; Nueangnoraj, K. Role of Nitrogen on the Porosity, Surface, and Electrochemical Characteristics of Activated Carbon. ACS Omega 2020, 5, 1911-1918.

(8) Gokce, Y.; Aktas, Z. Nitric acid modification of activated carbon produced from waste tea and adsorption of methylene blue and phenol. Appl. Surf. Sci. 2014, 313, 352-359.

(9) Menanteau, T.; Benoît, C.; Breton, T.; Cougnon, C. Enhancing the performance of a diazonium-modified carbon supercapacitor by controlling the grafting process. Electrochem. Commun. 2016, 63, 7073.

(10) Gao, F.; Qu, J.; Zhao, Z.; Wang, Z.; Qiu, J. Nitrogen-doped activated carbon derived from prawn shells for high-performance supercapacitors. Electrochim. Acta 2016, 190, 1134-1141.
(11) Sriprom, P.; Krusong, W.; Assawasaengrat, P. Preparation of Activated Carbon from Durian Rind with Difference Activations and Its Optimization. J. Renewable Mater. 2021, 9, 311-324.

(12) Sevilla, M.; Carro-Rodríguez, J.; Díez, N.; Fuertes, A. B. Straightforward synthesis of Sulfur/N,S-codoped carbon cathodes for Lithium-Sulfur batteries. Sci. Rep. 2020, 10, 4866.

(13) Torrellas, S. Á.; García Lovera, R.; Escalona, N.; Sepúlveda, C.; Sotelo, J. L.; García, J. Chemical-activated carbons from peach stones for the adsorption of emerging contaminants in aqueous solutions. Chem. Eng. J. 2015, 279, 788-798.

(14) Chandra, T. C.; Mirna, M. M.; Sunarso, J.; Sudaryanto, Y.; Ismadji, S. Activated carbon from durian shell: Preparation and characterization. J. Taiwan Inst. Chem. Eng. 2009, 40, 457-462.

(15) Yaglikci, S.; Gokce, Y.; Yagmur, E.; Aktas, Z. The performance of sulphur doped activated carbon supercapacitors prepared from waste tea. Environ. Technol. 2020, 41, 36-48.

(16) Roberts, A. D.; Li, X.; Zhang, H. Hierarchically porous sulfurcontaining activated carbon monoliths via ice-templating and one-step pyrolysis. Carbon 2015, 95, 268-278.

(17) Hao, E.; Liu, W.; Liu, S.; Zhang, Y.; Wang, H.; Chen, S.; Cheng, F.; Zhao, S.; Yang, H. Rich sulfur doped porous carbon materials derived from ginkgo leaves for multiple electrochemical energy storage devices. J. Mater. Chem. A 2017, 5, 2204-2214.

(18) Chen, H.; Yu, F.; Wang, G.; Chen, L.; Dai, B.; Peng, S. Nitrogen and Sulfur Self-Doped Activated Carbon Directly Derived from Elm Flower for High-Performance Supercapacitors. ACS Omega 2018, 3, 4724-4732.

(19) Liu, X.; Antonietti, M. Moderating black powder chemistry for the synthesis of doped and highly porous graphene nanoplatelets and their use in electrocatalysis. Adv. Mater. 2013, 25, 6284-6290.

(20) Zhao, B.; Xu, H.; Zhang, T.; Nan, X.; Ma, F. Effect of pyrolysis temperature on sulfur content, extractable fraction and release of sulfate in corn straw biochar. RSC Adv. 2018, 8, 35611-35617.

(21) Xu, L.; Yang, J.; Li, Y.; Liu, Z. Behavior of organic sulfur model compounds in pyrolysis under coal-like environment. Fuel Process. Technol. 2004, 85, 1013-1024.

(22) Song, Z.; Wang, M.; Batts, B. D.; Xiao, X. Hydrous pyrolysis transformation of organic sulfur compounds: Part 1 . Reactivity and chemical changes. Org. Geochem. 2005, 36, 1523-1532.

(23) Feng, W.; He, P.; Ding, S.; Zhang, G.; He, M.; Dong, F.; Wen, J.; Du, L.; Liu, M. Oxygen-doped activated carbons derived from three kinds of biomass: preparation, characterization and performance as electrode materials for supercapacitors. RSC $A d v$. 2016, 6, 59495956.

(24) Ariyanto, T.; Kurniasari, M.; Laksmana, W. T.; Prasetyo, I. Pore size control of polymer-derived carbon adsorbent and its application for dye removal. Int. J. Environ. Sci. Technol. 2019, 16, 4631-4636.

(25) Zhao, J.; Guan, B.; Ma, C.; Hu, B.; Zhang, H. Effect of elemental sulfur in precursors on the pore structure and surface chemical characteristics of high-surface area activated carbon. J. Saudi Chem. Soc. 2017, 21, 691-697.

(26) Fang, K.; Sheng, J.; Yang, R. Synthesis of Highly Microporous Sulfur-Containing Activated Carbons by a Multistep Modification Process. J. Wuhan Univ. Technol., Mater. Sci. Ed. 2020, 35, 856-862.

(27) Liu, S.; Cai, Y.; Zhao, X.; Liang, Y.; Zheng, M.; Hu, H.; Dong, H.; Jiang, S.; Liu, Y.; Xiao, Y. Sulfur-doped nanoporous carbon spheres with ultrahigh specific surface area and high electrochemical activity for supercapacitor. J. Power Sources 2017, 360, 373-382.

(28) White, J. L. Interpretation of Infrared Spectra of Soil Minerals. Soil Sci. 1971, 112, 22-31.

(29) Guo, Y.; Zeng, Z.; Liu, Y.; Huang, Z.; Cui, Y.; Yang, J. One-pot synthesis of sulfur doped activated carbon as a superior metal-free catalyst for the adsorption and catalytic oxidation of aqueous organics. J. Mater. Chem. A 2018, 6, 4055-4067.

(30) Shan, J.; Huang, J.-J.; Li, J.-Z.; Li, G.; Zhao, J.-T.; Fang, Y.-T. Insight into transformation of sulfur species during $\mathrm{KOH}$ activation of high sulfur petroleum coke. Fuel 2018, 215, 258-265. 
(31) Li, W.; Zhou, M.; Li, H.; Wang, K.; Cheng, S.; Jiang, K. A high performance sulfur-doped disordered carbon anode for sodium ion batteries. Energy Environ. Sci. 2015, 8, 2916-2921.

(32) Qie, L.; Chen, W.; Xiong, X.; Hu, C.; Zou, F.; Hu, P.; Huang, Y. Sulfur-Doped Carbon with Enlarged Interlayer Distance as a HighPerformance Anode Material for Sodium-Ion Batteries. Adv. Sci. 2015, 2,1500195 .

(33) Zhao, X.; Zhang, Q.; Chen, C.-M.; Zhang, B.; Reiche, S.; Wang, A.; Zhang, T.; Schlögl, R.; Sheng Su, D. Aromatic sulfide, sulfoxide, and sulfone mediated mesoporous carbon monolith for use in supercapacitor. Nano Energy 2012, 1, 624-630.

(34) Telfer, M.; Zhang, D.-K. The influence of water-soluble and acid-soluble inorganic matter on sulphur transformations during pyrolysis of low-rank coals. Fuel 2001, 80, 2085-2098.

(35) Weh, R.; de Klerk, A. Thermochemistry of Sulfones Relevant to Oxidative Desulfurization. Energy Fuels 2017, 31, 6607-6614.

(36) Guo, Z.; Fu, Z.; Wang, S. Sulfur distribution in coke and sulfur removal during pyrolysis. Fuel Process. Technol. 2007, 88, 935-941.

(37) Elmouwahidi, A.; Castelo-Quibén, J.; Vivo-Vilches, J. F.; PérezCadenas, A. F.; Maldonado-Hódar, F. J.; Carrasco-Marín, F. Activated carbons from agricultural waste solvothermally doped with sulphur as electrodes for supercapacitors. Chem. Eng. J. 2018, 334, 1835-1841. (38) Ji, H.; Wang, T.; Liu, Y.; Lu, C.; Yang, G.; Ding, W.; Hou, W. A novel approach for sulfur-doped hierarchically porous carbon with excellent capacitance for electrochemical energy storage. Chem. Commun. 2016, 52, 12725-12728.

(39) Wang, T.; Wang, L. X.; Wu, D. L.; Xia, W.; Jia, D. Z. Interaction between nitrogen and sulfur in co-doped graphene and synergetic effect in supercapacitor. Sci. Rep. 2015, 5, 9591.

(40) Guo, Y.; Zeng, Z.; Li, Y.; Huang, Z.; Yang, J. Catalytic oxidation of 4-chlorophenol on in-situ sulfur-doped activated carbon with sulfate radicals. Sep. Purif. Technol. 2017, 179, 257-264.

(41) Zhang, J.; Yang, Z.; Wang, X.; Ren, T.; Qiao, Q. Homogeneous sulphur-doped composites: porous carbon materials with unique hierarchical porous nanostructure for super-capacitor application. RSC Adv. 2016, 6, 84847-84853.

(42) Dubinin, M. M. Physical Adsorption of Gases and Vapors in Micropores. In Progress in Surface and Membrane Science; Cadenhead, D.A.; Danielli, J. F.; Rosenberg, M. D., Eds. Academic Press, Inc.: New York, 1975; Vol. 9, pp. 1-70. 